\title{
UNA EXPERIENCIA DE ACOMPAÑAMIENTO CON DOULA A ADOLESCENTES EN TRABAJO DE PARTO
}

\author{
Edda Pugin P. ${ }^{1 a}$, Erika Kopplin I. ${ }^{1 b}$, Carmen Larraín C. ${ }^{1 c}$, Verónica Gallego J. ${ }^{2 a}$, \\ Marco Aramayo R. ${ }^{2}$, Juan Ortiz C. ${ }^{2}$ \\ ${ }^{1}$ Programa Emprende Mamá. ${ }^{2}$ Servicio de Obstetricia y Ginecología, Hospital Dr. Sótero del Río. \\ a Matrona. ${ }^{b}$ Psicóloga. ${ }^{c}$ Directora del Programa
}

\section{RESUMEN}

Objetivo: Comparar la percepción del dolor-temor durante el trabajo de parto y parto, y el grado de dificultadsatisfacción con la experiencia de parto, en un grupo de adolescentes acompañadas por doula y otro con atención habitual. Método: El grupo estudio comprendió 80 adolescentes del "Programa Emprende Mamá" que controlaron sus embarazos en centros de salud de la comuna de La Florida y con parto en el hospital Dr. Sótero del Río, acompañadas por una mujer familiar o amiga, elegida por la joven, con capacitación previa de 2 horas. El grupo control estuvo formado por 80 adolescentes no participantes del programa, que controlaron sus embarazos en los mismos centros de salud, con parto en el mismo período. Resultados: El grupo estudio refirió menor percepción de dolor durante el trabajo de parto y parto, menor temor durante el parto y lo percibió como más fácil. No se encontraron diferencias significativas en el nivel de satisfacción respecto de la experiencia total. Sin embargo, las adolescentes con doula señalaron estar satisfechas por aspectos positivos de la vivencia, mientras que el grupo control centra su satisfacción en la ausencia de expectativas negativas temidas. Conclusión: El acompañamiento por doula en el parto de la embarazada adolescente, es una intervención de bajo costo que permite mejorar cualitativamente su vivencia de parto.

\section{PALABRAS CLAVE: Doula, afectividad, apoyo emocional, embarazo adolescente}

\section{SUMMARY}

Objective: To compare the effects of continuous doula support and standard hospital care on pain and fear perception, during labor and delivery, as well as perception of difficulty and satisfaction with the birth experience, in a sample of pregnant teenagers. Method: The study group was conformed by eighty pregnant teenagers participants in "Emprende Mamá Program" attended in the outpatient clinics of La Florida county for their prenatal care and delivery at a public hospital. They received emotional support during labor by a 2 hour trained female relative or friend, previously chosen by them. The control group was conformed by eighty non participants teenagers attended at the same outpatient clinics and delivery in the same period. Results: Teenagers with doula support reported less pain and fear during labor and delivery and perceived their birth experience as less difficult. No significant differences were reported in relation to satisfaction with birth experience. Although teenagers with doula support reported satisfaction due to positive aspects with birth experience, meanwhile the control group felt mostly satisfied due the absence of negative feared expectations. Conclusion: Labor support given by doula, is a low cost intervention which improves quality perception of birth experience in the adolescent pregnant.

KEY WORDS: Doula, affectivity, emotional support, adolescent pregnancy 


\section{INTRODUCCIÓN}

Diversos estudios señalan los beneficios del apoyo afectivo durante el trabajo de parto. Este se define como la presencia de una acompañante capacitada y con experiencia que proporciona a la mujer y su pareja, apoyo emocional continuo, confort físico y asistencia para obtener información antes, durante y después del parto, llamada doula.

Diversos meta-análisis confirman las ventajas de esta práctica tales como: reducción de la duración del trabajo de parto, necesidad de analgesia y de cesáreas (1-4), menor necesidad de hospitalización del recién nacido y mayor duración de lactancia materna $(5,6)$. Otros estudios señalan importantes efectos sicológicos en la mujer como, menor temor al momento del parto (7) y mayor grado de satisfacción con la experiencia de parto (1). Entre los efectos a largo plazo se cuentan una mayor seguridad para desempeñar su rol de madre y mejor autoestima (8-10). Esta evidencia científica avala la necesidad de recuperar este componente afectivo, que permitió la sobrevivencia de nuestra especie y combinarlo en la justa proporción con los avances científicos que nos han permitido mejorar nuestra calidad de vida.

Desde el 2001 se desarrolla en la comuna de La Florida (Santiago, Chile) el programa "Emprende Mamá", dirigido a adolescentes embarazadas de bajos recursos (11). En esta intervención se extiende el acompañamiento desde la gestación al primer año de vida del hijo, considerando los factores de riesgo asociados al embarazo adolescente (12-16). Fue sistematizado el 2005 en un proyecto de investigación-acción solicitado por MIDEPLAN, e incorporado el 2007 al Sistema de Protección a la Infancia del Gobierno de Chile denominado "Chile Crece Contigo" (17), en la comuna de La Florida. En un trabajo coordinado con la maternidad del hospital Dr. Sótero del Río, desde el año 2003, se incorporó una doula (mujer familiar o amiga) durante el trabajo de parto, elegida por la adolescente y capacitada previamente por el equipo profesional. Esta modalidad ha sido actualmente descrita como de bajo costo, alta efectividad y contribuyente a favorecer el bienestar materno infantil (18).

El objetivo de este estudio es comparar los aspectos cualitativos de la experiencia de parto, en una muestra de adolescentes del programa, que tuvieron su parto acompañado por doula con un grupo similar que no lo tuvo.

\section{MATERIAL Y MÉTODO}

El diseño metodológico fue de tipo cuasi experi- mental sin medición previa. La muestra total estuvo formada por 160 madres adolescentes participantes del programa y madres adolescentes no participantes, todas usuarias de centros de salud de la comuna de La Florida. El grupo estudio se extrajo de un total de 158 adolescentes que tuvieron doula capacitada y que participaron en el programa entre los años 2003 y 2006. De esa población se seleccionó a aquellas que cumplieron con las siguientes condiciones: haber tenido parto normal (requeridos por el hospital para permitir la entrada de la doula) y haber estado acompañada durante el trabajo de parto por doula. Entre enero y diciembre de 2006, 80 adolescentes de un total de 95 pudieron ser contactadas telefónicamente, para la aplicación de la encuesta y que cumplían con los requisitos señalados (Tabla I).

\section{Tabla I}

CARACTERÍSTICAS DE LAS ADOLESCENTES DE LOS GRUPOS ESTUDIO Y CONTROL

\begin{tabular}{ccc}
\hline Variables & $\begin{array}{c}\text { Grupo estudio } \\
(n=80)\end{array}$ & $\begin{array}{c}\text { Grupo control } \\
(n=80)\end{array}$ \\
\hline
\end{tabular}

Edad en años:

$\begin{array}{lll}13-15 & 20 \% & 21,3 \% \\ 16-18 & 60 \% & 67,5 \% \\ 19 & 20 \% & 11,3 \%\end{array}$

Situación de pareja:

$\begin{array}{lrr}\text { Con apoyo } & 92,5 \% & 90 \% \\ \text { Sin apoyo } & 7,5 \% & 10 \%\end{array}$

Edad del hijo:

$\begin{array}{lll}<2 \text { años } & 65 \% & 62,5 \% \\ 2 \text { a } 4 \text { años } & 35 \% & 37,5 \%\end{array}$

Tipo de parto:

$\begin{array}{lll}\text { Vaginal } & 83,8 \% & 83,8 \% \\ \text { Vaginal fórceps } & 16,2 \% & 16,2 \%\end{array}$

Atención en maternidad:

$\begin{array}{lll}\text { Buena } & 90 \% & 87,5 \% \\ \text { Regular o mala } & 10 \% & 12,5 \%\end{array}$

Apego realizado:

$\begin{array}{lll}\text { Si } & 67,9 \% & 66,2 \% \\ \text { No } & 32,1 \% & 33,8 \%\end{array}$


El grupo control estuvo formado por madres adolescentes no participantes del programa, entrevistadas en los centros de salud entre los meses de septiembre y noviembre de 2006. Los criterios de inclusión fueron: no haber participado en el programa ni otro similar, 19 años ó menos al momento del embarazo, parto normal, primer hijo y edad de éste entre 6 meses y 4 años al momento de la encuesta.

Para hacer comparables ambos grupos se consideraron algunas variables que pudieran afectar los resultados en forma independiente a la intervención realizada. Estas variables fueron: edad de la adolescente al momento del embarazo en rangos, edad del hijo al momento de aplicación del cuestionario en rangos, uso de fórceps, apoyo afectivo de la pareja, calidad de la atención percibida (en escala 1 a 7) y apego realizado. Respecto a éste, se considera que existe si las madres permanecen la primera hora después de nacido con su hijo en contacto piel a piel. La mayoría reportó contacto inicial con su hijo menor a 15 minutos. Se consideró por tanto apego el sólo hecho de permitirles contacto piel a piel durante unos minutos.

De 93 entrevistadas que cumplieron los criterios, se seleccionaron al azar 80 , buscando reproducir distribución similar al grupo de estudio en las variables intervinientes, lográndose al nivel total de cada variable, de modo que no hubo diferencias significativas entre ambos grupos en ninguna de éstas (Tabla II). La mayoría de las adolescentes del grupo control tuvo su parto en el hospital Dr. Sótero del Río $(68,8 \%)$. El resto se distribuyó entre hospital Dr. Luis Tizné (5\%), hospital Alberto Hurtado $(8,8 \%)$, clínicas privadas $(5 \%)$ y otros.
Las variables dependientes fueron:

1.Dolor percibido durante el trabajo de parto, evaluado con escala visual análoga EVA (19), de 0 a 10, siendo 10 el máximo de dolor experimentado.

2.Dolor percibido durante el parto, con la misma escala.

3.Temor experimentado al momento del parto, medido con escala de 0 a 10 puntos.

4.Grado de dificultad percibido en el parto, a través de escala tipo Likert de 5 alternativas: muy difícil, difícil, regular, fácil o muy fácil.

5.Satisfacción con la experiencia de parto, en escala Likert con 5 alternativas: Muy satisfecha, satisfecha, regularmente satisfecha, insatisfecha y muy insatisfecha.

Las 3 primeras variables fueron dicotomizadas, para algunos análisis en 2 categorías: bajo nivel de dolor o temor (puntaje 0 a 5) y alto nivel de dolor o temor (puntaje 6 a 10). Las 2 últimas variables fueron dicotomizadas en 2 categorías: alto nivel de dificultad versus regular o bajo, y alto nivel de satisfacción versus regular o bajo.

Las acompañantes fueron capacitadas grupalmente previo al parto por la matrona y psicóloga del programa en relación a apoyo emocional y uso de medidas de confort durante el trabajo de parto (masaje, visualización y respiración, entre otras), en talleres de 2 horas de duración. Mayoritariamente la doula fue la madre $(72 \%)$, seguidas por suegra (8\%) y tía (6\%). Para el análisis estadístico se utilizó la prueba de diferencia de promedios t de Student, análisis de varianza y prueba de chi cuadrado. En algunos casos se calculó la correlación lineal de Pearson. Se consideró significativo un $\mathrm{p}<0,05$.

Tabla II

COMPARACIÓN SEGÚN VARIABLES DE CRITERIO ANALIZADO ENTRE GRUPOS ESTUDIO Y CONTROL

\begin{tabular}{lccc}
\hline Variables intervinientes & $\begin{array}{c}\text { Grupo estudio } \\
\mathrm{n}=80\end{array}$ & $\begin{array}{c}\text { Grupo control } \\
\mathrm{n}=80\end{array}$ & Valor $\mathrm{p}$ \\
\hline Edad promedio en años & 16,9 & 17 & $\mathrm{NS}$ \\
Hijo menor de 2 años & $65 \%$ & $63 \%$ & $\mathrm{NS}$ \\
Presencia de pareja & $90 \%$ & $93 \%$ & $\mathrm{NS}$ \\
Parto vaginal fórceps & $16 \%$ & $16 \%$ & $\mathrm{NS}$ \\
Atención en maternidad * & $90 \%$ & $88 \%$ & $\mathrm{NS}$ \\
Hubo apego & 67,9 & 66,2 & $\mathrm{NS}$ \\
\hline
\end{tabular}

\footnotetext{
${ }^{*}$ : buena o muy buena. NS: no significativo.
} 


\section{RESULTADOS}

Hubo diferencias significativas a favor del grupo con doula en 4 de las 5 variables estudiadas (Tablas III y IV). Las adolescentes acompañadas por doula tuvieron menor percepción de dolor durante el trabajo de parto, menor percepción de dolor du- rante el parto, menos temor al momento del parto y una percepción del parto más fácil que difícil, comparadas con las adolescentes que no tuvieron acompañamiento. Posteriormente, se elaboró un índice acumulativo que incluía las variables "dolor percibido durante el trabajo de parto", "dolor percibido en el parto" y "temor experimentado durante

Tabla III

\section{RESULTADOS EN PUNTAJES PROMEDIOS DE VARIABLES ESTUDIADAS SEGÚN GRUPOS ESTUDIO Y CONTROL}

\begin{tabular}{lcccc}
\hline Variables & Grupo estudio & Grupo control & $\mathrm{t}$ & Valor $\mathrm{p}$ \\
\hline Dolor percibido en trabajo de parto & 6,2 & 7,9 & $-3,42$ & 0,00 \\
Dolor percibido durante el parto & 2,4 & 3,6 & $-2,08$ & 0,004 \\
Temor durante el parto & 3,2 & 6 & $-4,87$ & 0,00 \\
Índice acumulativo de dolor y temor & 11,8 & 17,5 & $-5,26$ & 0,00 \\
\hline
\end{tabular}

Tabla IV

RESULTADOS EN CATEGORÍAS DE VARIABLES ESTUDIADAS SEGÚN GRUPOS ESTUDIO Y CONTROL

\begin{tabular}{|c|c|c|c|}
\hline Variables & Grupo estudio & Grupo control & Valor $p$ \\
\hline Dolor percibido en trabajo de parto: & & & 0,00 \\
\hline Bajo dolor $(0-5)$ & $40 \%$ & $18,8 \%$ & \\
\hline Alto dolor $(6-10)$ & $60 \%$ & $81,2 \%$ & \\
\hline Dolor percibido durante el parto: & & & NS \\
\hline Bajo dolor $(0-5)$ & $81,2 \%$ & $71,2 \%$ & \\
\hline Alto dolor $(6-10)$ & $18,8 \%$ & $28,8 \%$ & \\
\hline Temor durante el parto: & & & 0,00 \\
\hline Bajo nivel de temor & $73,8 \%$ & $45 \%$ & \\
\hline Alto nivel de temor & $26,2 \%$ & $55 \%$ & \\
\hline Índice acumulativo de dolor y temor: & & & 0,00 \\
\hline Bajo puntaje & 73,8 & 41,3 & \\
\hline Alto puntaje & 26,3 & 58,8 & \\
\hline Dificultad percibida del parto: & & & 0,00 \\
\hline Fácil o muy fácil & $55 \%$ & $35 \%$ & \\
\hline Regular, difícil o muy difícil & $45 \%$ & $65 \%$ & \\
\hline Satisfacción con experiencia de parto: & & & NS \\
\hline Muy satisfecha & 51,3 & 32,5 & \\
\hline Satisfecha & 42,5 & 53,8 & \\
\hline Regularmente satisfecha & 3,8 & 7,5 & \\
\hline Insatisfecha o muy insatisfecha & 2,6 & 6,3 & \\
\hline
\end{tabular}

NS: no significativo. 
el parto". Este tuvo un rango entre 0 y 30 , considerando que cada variable incluida fluctuaba entre 0 y 10 , indicando una experiencia más insatisfactoria a medida que aumenta el valor. Este índice mostró diferencias significativas entre ambos grupos, a favor de las adolescentes acompañadas, mostrando éstas menor dolor y temor durante la experiencia de nacimiento de su hijo. En el grupo de estudio el índice se correlacionó más con el dolor percibido durante el parto $(r=0,75)$ y el dolor percibido durante el trabajo de parto $(r=0,73)$, mientras que en el grupo control se asocia con dolor percibido durante el parto $(r=0,68)$ y con temor experimentado $(r=0,65)$.

El grado de dificultad del parto se asoció en el grupo estudio principalmente con la percepción de dolor durante el trabajo de parto y el parto mismo. El parto se percibió más fácil en la medida que el dolor fue menor, con la percepción de dolor durante el trabajo de parto $(C$ real $=0,39 ; p=0,03$ ) y dolor durante el parto $(C$ real $=0,40 ; p=0,04)$. En el grupo control en cambio, sólo el dolor experimentado durante el parto mismo se asoció significativamente con la percepción de facilidad o dificultad del parto $(C$ real $=0,52 ; p=0,00)$.

La satisfacción con la experiencia de parto, no resultó estadísticamente diferente entre el grupo de estudio y el grupo control. Así, el 94 y $86 \%$ de las adolescentes, respectivamente, reveló estar satisfecha o muy satisfecha con la experiencia de parto. Sólo podría decirse que existe una leve tendencia a ser mayor en el grupo estudio, dado que las adolescentes de este grupo muestran en mayor proporción estar "muy satisfechas", mientras la categoría modal del grupo control es "satisfechas".

Al analizar la posible influencia de la percepción de dolor con el grado de satisfacción, se encontró que en el grupo de estudio no hubo una asociación significativa entre la satisfacción con la experiencia de parto, y el dolor durante el trabajo de parto o durante el parto mismo ni el temor experimentado. En el grupo control, en cambio, el dolor experimentado durante el parto se asocia más claramente con el grado de satisfacción ( $C$ real $=0,47 ; p=0,01$ ),

Las razones que dieron las adolescentes del grupo estudio para evaluar como alta o muy alta su satisfacción con esta experiencia, aludieron más notoriamente que las del grupo control a la experiencia positiva del parto y al hecho de poder estar acompañada de un ser querido. Las adolescentes del grupo control, en cambio la atribuyeron mayoritariamente a haber tenido un hijo sano y que no sufrieron tanto dolor. Por su parte, las razones más frecuentes que dieron las adolescentes del grupo estudio para evaluar como regular o baja su satis- facción, fue lo largo del trabajo de parto o el parto mismo. En cambio las razones del grupo control fueron relativas al dolor y a la falta de apego hecho en el hospital.

Al analizar el efecto de las variables intervinientes sobre las variables independientes, se observó que el apoyo de la pareja incide en disminuir la percepción de dolor en el parto, tanto en el grupo de estudio como en el grupo de control. En relación a la edad, las adolescentes de mayor edad del grupo de estudio tienen puntajes más altos de dolor percibido en el parto. En cambio la edad afecta en sentido contrario, es decir, mientras menor la edad de la adolescente mayor es la percepción de dolor durante el parto el grupo control.

Al realizar un modelo lineal general incluyendo como variables independientes el grupo, la edad y el apoyo de la pareja, se aprecia una diferencia significativa entre ambos $(t=2,50 ; p=0,01)$, es decir, la presencia de una doula logra disminuir la percepción de dolor durante el parto independientemente de la edad y el apoyo de la pareja. A su vez al evaluar la influencia del apoyo afectivo de la pareja sobre el índice acumulativo, se vio que en el grupo control, las adolescentes que contaron con éste durante el embarazo mostraron menor puntaje en este índice, tendencia que no se apreció en el grupo estudio, es decir, nuevamente en el grupo acompañado no influyó la ausencia de apoyo de la pareja en la vivencia de la experiencia de parto.

Con respecto a las variables intervinientes en el grupo de estudio, el tipo de parto y la atención recibida en la maternidad influyen en la satisfacción. Así, se mostraron más satisfechas las que tuvieron un parto sin fórceps ( $C$ real $=0,50 ; p=0,00$ ) y las que evaluaron la atención como buena $(C$ real $=0,62 ; p=$ $0,00)$. En el grupo control la atención recibida en la maternidad fue la única variable interviniente que influyó en la satisfacción ( $C$ real $=0,55, p=0,00$ ).

Finalmente, se destaca que ante una pregunta realizada a las adolescentes del grupo control, el $68 \%$ de éstas señala que le hubiera gustado estar acompañadas por alguna mujer familiar durante este proceso. A su vez, la mayoría de las jóvenes acompañadas (85\%) señalaron que el aspecto más importante en su trabajo de parto fue la presencia o compañía de la doula, por sobre otras medidas de confort recibidas.

\section{DISCUSIÓN}

Los resultados de este estudio apoyan la creciente evidencia respecto a que el apoyo emocional continuo durante el trabajo de parto, tiene efectos positivos, no sólo en aspectos fisiológicos de éste, 
sino también sobre algunos aspectos cualitativos de la experiencia.

La presencia de doula resultó efectiva, logrando que las adolescentes disminuyan significativamente su percepción de dolor y temor en el trabajo de parto y parto, y que perciban su parto como más fácil, en comparación a una población de similar edad que no tuvo acompañamiento. Esta diferencia se mantuvo al agrupar las variables antes señaladas en un índice acumulativo de dolor y temor, por lo que se puede concluir que el acompañamiento ha sido efectivo en disminuir la percepción de dolor y temor en la experiencia del parto de las adolescentes. Este efecto fue especialmente notorio en aquellas jóvenes de menor edad y las que no contaban con el apoyo afectivo de su pareja, es decir, el grupo más vulnerable.

Por otro lado, el acompañamiento recibido no tuvo un efecto significativo en la evaluación final de satisfacción de la experiencia al comparar ambos grupos, ya que ésta estuvo más influida por la atención recibida por parte del equipo de maternidad. Sin embargo, cualitativamente se observaron diferencias en las causas por las que las jóvenes madres puntúan su experiencia como satisfactoria o muy satisfactoria. Mientras el grupo estudio se centra en aspectos positivos de la vivencia misma, el grupo control lo hace respecto a la ausencia de expectativas negativas temidas.

En este estudio se vio que las adolescentes valoran estar acompañadas por alguien con quien existe un lazo afectivo previo, lo que queda demostrado por el alto porcentaje de adolescentes del grupo estudio que valoran la presencia y compañía de un familiar conocido por sobre las medidas de confort ofrecidas por éstas durante el pre-parto, y el alto porcentaje de adolescentes controles que les hubiera gustado la compañía de un familiar femenino durante el trabajo de parto.

Es importante señalar que no fue posible aislar sólo el efecto del acompañamiento con doula durante el trabajo de parto, del resto de la intervención recibida por el grupo estudio, como es el acompañamiento psico-afectivo y educativo recibido durante la gestación, de modo que los resultados dan cuenta de los efectos sumatorios de acompañamiento tanto durante el embarazo como en el pre-parto.

\section{CONCLUSIÓN}

Estos hallazgos estimulan iniciativas para proveer doulas mínimamente capacitadas, idealmente con lazo afectivo previo con la gestante, como alternativa para incorporar el apoyo emocional en la experiencia del nacimiento, lo que simplemente significa volver a las tradiciones ancestrales. Esto es aún más necesario cuando se trata de un grupo de riesgo como es la gestante adolescente, por su vulnerabilidad psico-social por la temprana edad con que inician la maternidad. Esta experiencia demuestra que es posible realizar una intervención de bajo costo en un hospital público, que ayuda a personalizar el proceso del nacimiento.

RECONOCIMIENTO: Los aspectos metodológicos de diseño muestral y análisis de datos fueron financiados por Fosis como parte de la investigación- acción "Sistematización Programa Emprende Mamá" y realizado por la evaluadora externa Sra. Valeria Ramírez, socióloga y docente de la Pontificia Universidad Católica de Chile.

\section{BIBLIOGRAFÍA}

1. Hodnett ED, Gates S, Hofmeyr GJ, Sakala C. Continuous support for women during childbirth. Cochrane database of Systematic Review 2007, Issue Oct.18;(3): CD003766.

2. Zhang J, Bernasko JW, Leybovich E, Fahs M, Hatch MC. Continuous labor support from labor attendant for primiparous women: a meta-analysis. Obstet Gynecol 1996;88(4 Pt 2):739-44.

3. Scott KD, Berkowitz G, Klaus M. A comparison of intermittent and continuous support during labor: a metaanalysis. Am J Obstet Gynecol 1999;180(5):1054-9.

4. Klaus $\mathrm{MH}$, Kennell $\mathrm{JH}$. The doula: an essential ingredient of childbirth rediscovered. Acta Paediatr 1997;86(10):1034-6.

5. Hofmeyer GJ, Nykodem VC, Wolman W, et al. Companionship to modify the clinical birth environment: Effect on progress and perceptions of labor and breastfeeding. Br Obstet Gynecol 1991;98:756-64.

6. Langer A, Campero L, García C, Reinoso S. Effects of psychosocial support during labour and childbirth on breastfeeding, medical interventions and mothers' wellbeing in a Mexican public hospital: a randomized clinical trial. Br J Obstet Ginaecol 1998;105(10):105663.

7. Torres J, Kopplin E, Peña V, Klaus M, Salinas R, Herrera M. Impacto del apoyo emocional durante el parto en la disminución de cesáreas y gratificación del proceso. Rev Chil Obstet Ginecol 1999;64(5):405-12.

8. Gordon NP, Walton D, McAdam E, Derman J, Gallitero G, Garrett L. Effects of providing hospital-based doulas in health maintenance organization hospitals. Obstet Gynecol 1999;93(3):422-6.

9. Landry SH, Mc Grath S, Kennell JH, Martin S, Steelman L. The effect of doula support during labor on mother-infant interaction at 2 months. Pediatr Res 1998;43(4):Supp 2:13.

10. Valdés $V$, Morlans $X$. Aportes de las doulas a la obstetricia moderna. Rev Chil Obst Ginecol 2005;70(2):10812.

11. Cabezas, AM, Kopplin E, Pugin E, Larraín C, Palma $\mathrm{P}$, González M. Programa de acompañamiento psicoafectivo a adolescentes embarazadas en riesgo social. Pediatría al Día 2003;19(5):41-5. 
12. Molina R. Salud sexual y reproductiva en la adolescencia. Editorial Mediterráneo, Santiago, 2003.

13. Alatorre Rico J, Lucile C. El embarazo adolescente y la pobreza. En: Bonfil P, Vania S (eds.). Mujeres pobres: Salud y trabajo. México: Gimtrap 1998. pp. 1330.

14. Buvinic M, Valenzuela JP, Molina T, González E. La suerte de las madres adolescentes y sus hijos: Un estudio de casos sobre la transmisión de pobreza en Santiago de Chile. Cepal 1991, Documento LC/ R1038.

15. Kopplin E. Aspectos psicosociales de la embarazada y madre adolescente. En: Compromisos antes de tiempo: adolescentes, sexualidad y embarazo, Publicación Corsaps, Santiago, Corporación de Salud y Políticas Sociales, 1989: 49-63.

16. Musick J. Young, poor, and pregnant: the psychology of teenage motherhood. Yale University Press, 1993.

17. Sistema de Protección Integral a la Primera Infancia, Chile Crece Contigo, Gobierno de Chile. Hallado en: www.crececontigo.cl

18. Campbell D, Scott K, Klaus M, Falk M. Female relatives or friends trained as labor doulas: outcomes at 6 to 8 weeks postpartum. Birth 2007;34(3):220- 7 .

19. Huskisson EC. Measurement of pain. Lancet 1974;2:1127-31. 